\title{
Diseases of the Colon and Rectum: CT Colonography
}

\author{
C. Dan Johnson and Perry J. Pickhardt
}

\section{Learning Objectives}

- To implement proper technique for obtaining a high-quality CT colonography examination

- To educate others regarding the safety and performance of CT colonography

- To participate in a quality improvement program related to CT colonography

- To recognize difficult-to-detect lesions at CT colonography

The public health need for colorectal cancer screening is compelling. Colorectal cancer is common, accounting for approximately 50,000 deaths yearly in the USA [1]. The benign precursor adenoma can be detected by several different imaging techniques, and, if removed, can prevent malignant transformation. The approximately 10-year polyp dwell time allows ample opportunity for patients to be screened and polyps detected and removed. Potentially, under ideal screening circumstances, an entire class of cancers could be prevented. Unfortunately, barriers exist to ideal screening, including suboptimal performance of many existing colorectal screening tests, reluctant compliance by patients to follow recommended screening guidelines, and variable insurance coverage of examination charges. In many ways, CT colonography (CTC) approaches an ideal screening test by addressing issues and problems inherent with other techniques. This syllabus will highlight many key issues for CT colonography today.

C. Dan Johnson, M.D. (ه)

Mayo Clinic, Scottsdale, AZ, USA

e-mail: johnson.daniel2@mayo.edu

P. J. Pickhardt, M.D.

University of Wisconsin, Madison, WI, USA

\subsection{Technique}

Proper technique at CT colonography is imperative for high performance. The three main steps include preparation, CT scanning, and interpretation.

\subsubsection{Preparation}

1. Dietary restriction. Preparation begins with a recommendation for patients to consume clear liquids or a lowresidue diet for at least 1 day prior to beginning the cathartic bowel preparation. Although this step is optimal, it can be skipped if patients are following the subsequent instructions carefully.

2. Stool tagging. 10-20 mL of barium or iodinated contrast material is administered orally for three meals prior to beginning the cathartic preparation. This contrast agent mixes with stool and ideally is evacuated with the cathartic preparation. Residual stool will contain highattenuation contrast and can be easily discriminated from soft tissue attenuation polyps. Some prefer to perform stool tagging after the cathartic agent, but before fluid tagging.

3. Cathartic. The cathartic preparation can be performed with a number of different formulations including polyethylene glycol electrolyte solution and magnesium citrate or bisacodyl tablets. Phospho-soda agents are not recommended because of prior issues with renal insufficiency and sodium retention. Bisacodyl tablets require the patient to be well hydrated for the purgation process to be successful. Bisacodyl tablets are easy to consume and well tolerated by patients. Polyethylene glycol electrolyte solution provides the cleanest colon, but some patients find it more difficult to consume in its entirety.

4. Fluid tagging. Following the cathartic preparation, the small bowel will continue to secrete soft tissue attenuation fluid into the colon. This fluid can obscure immersed pol- 
yps. Tagging of this fluid is accomplished by drinking $60 \mathrm{ml}$ of iodinated contrast material (either hyperosmolar or iso-osmolar) at bedtime, on the day before the exam. The high attenuation tagging allows inspection of the immersed portions of the colon wall and visualization of any colon lesions within this fluid.

5. Insufflation. The colon must be fully distended with air or carbon dioxide for optimal visualization at CT. Commercially available insufflation devices are available instilling $\mathrm{CO}_{2}$ at a predefined rate and pressure. Excess intraluminal colonic pressure during a colonic contraction will result in venting of gas into the room - thereby reducing patient discomfort and the risk of perforation. As the pressure is reduced, the machine automatically refills the colon. Turning the patient on the CT table is helpful in creating a well-distended colon.

6. Spasmolytics [2]. The use of spasmolytics is controversial. Although it has not been shown to improve image quality, there are some that believe the exam is more comfortable for patients [3].

\subsubsection{CT Scanning}

Acquisition of CT data using thin collimation and isotropic reconstruction intervals will result in high-quality images in any desired plane. The colon and rectum data should be acquired in a single breath-hold, using a low-dose technique. Generally, we use a $1 \mathrm{~mm}$ collimation, reconstructing every $0.8 \mathrm{~mm}, 50 \mathrm{mAs}$. Optimally, new reconstruction software should be employed to enable ultralow-dose acquisition. Ideally, average patients should receive an effective dose equivalent of less than $5 \mathrm{mSv}$ [4].

\subsubsection{Interpretation}

There are two main ways to interpret a CTC examination: either a primary $2 \mathrm{D}$ with $3 \mathrm{D}$ problem-solving or primary $3 \mathrm{D}$ with $2 \mathrm{D}$ problem-solving approaches. Both methods have been shown to have equal effectiveness. Most authorities advocate that each exam should have both a 2D and 3D review. Small polyps are more easily detected with a 3D (endoluminal) review, whereas obstructing cancers and flat lesions are better detected with a $2 \mathrm{D}$ review. There is no proven difference between software manufacturers in lesion detection [5] (Fig. 16.1).

\subsection{Performance}

The performance of CT colonography has undergone exhaustive testing. The Department of Defense (DoD) trial by Pickhardt et al. demonstrated sensitivity similar to colonoscopy [6], but concerns were raised that community practices might not be able to achieve these results. The National CT Colonography Trial findings were similar to the Pickhardt's trial and have reassured many groups [7] that the test can be performed with high accuracy in both academic and private practice settings.

Training and preferably testing of radiologists are requirements for optimal reader performance [8]. Participation in a dedicated training program is recommended. These training sessions should provide enough time for the radiologist to become facile with a specific colonography software package and experience interpreting at least 50 proven cases. Polyp detection testing will allow individuals an opportunity to assess if additional training is needed before clinical implementation. In order to continue to improve reader performance, it is recommended that patients who are undergoing both CTC and subsequent colonoscopy be reviewed retrospectively to assess for CTC false-positive and false-negative detections. This quality improvement review offers a rich experience for learning and gaining expertise, confidence, and competence. Strict adherence to state-of-the art CTC protocol requirements is also recommended including stool and fluid tagging regimens, mechanical insufflation of the colon, thinsection data acquisition, and low-dose CT techniques [9]. It is clear that meticulous attention to all aspects of the examination and interpretation is required to achieve the best results.

\subsection{Acceptance}

Today, nearly 40 million US adults age 50 or older have not undergone a sigmoidoscopy or colonoscopy within the previous 10 years or had used a fecal occult blood test (FOBT) home test kit within the preceding year [10]. The major disincentive for patients undergoing a full structural colorectal examination is the laxative purgation [11]. Work is being done to reduce the burden of laxation either with the use of a partial colon preparation [12] or without cathartic preparation [13]. Although not considered standard of care, evidence from feasibility trials is promising and may translate to better patient acceptance in the future.

Despite a full cathartic bowel preparation, the advantages of CTC include the lack of required sedation and intravenous line placement, a quick return to work following the examination, and the convenience of no driving restrictions following the test.

The disadvantage of CTC includes the fact that if a significant polyp is identified, patients must undergo colonoscopy and polypectomy. If same-day colonoscopy is not offered, then the patient must undergo a second bowel preparation, spend another day away from work, and experience added worry and inconvenience. In a screening population (including false-positive interpretations), the prevalence of patients being referred from CTC to colonoscopy for polypectomy is $12 \%$ when a $6 \mathrm{~mm}$ threshold is applied [6, 7]. This translates into an $88 \%$ chance that an individual patient will 

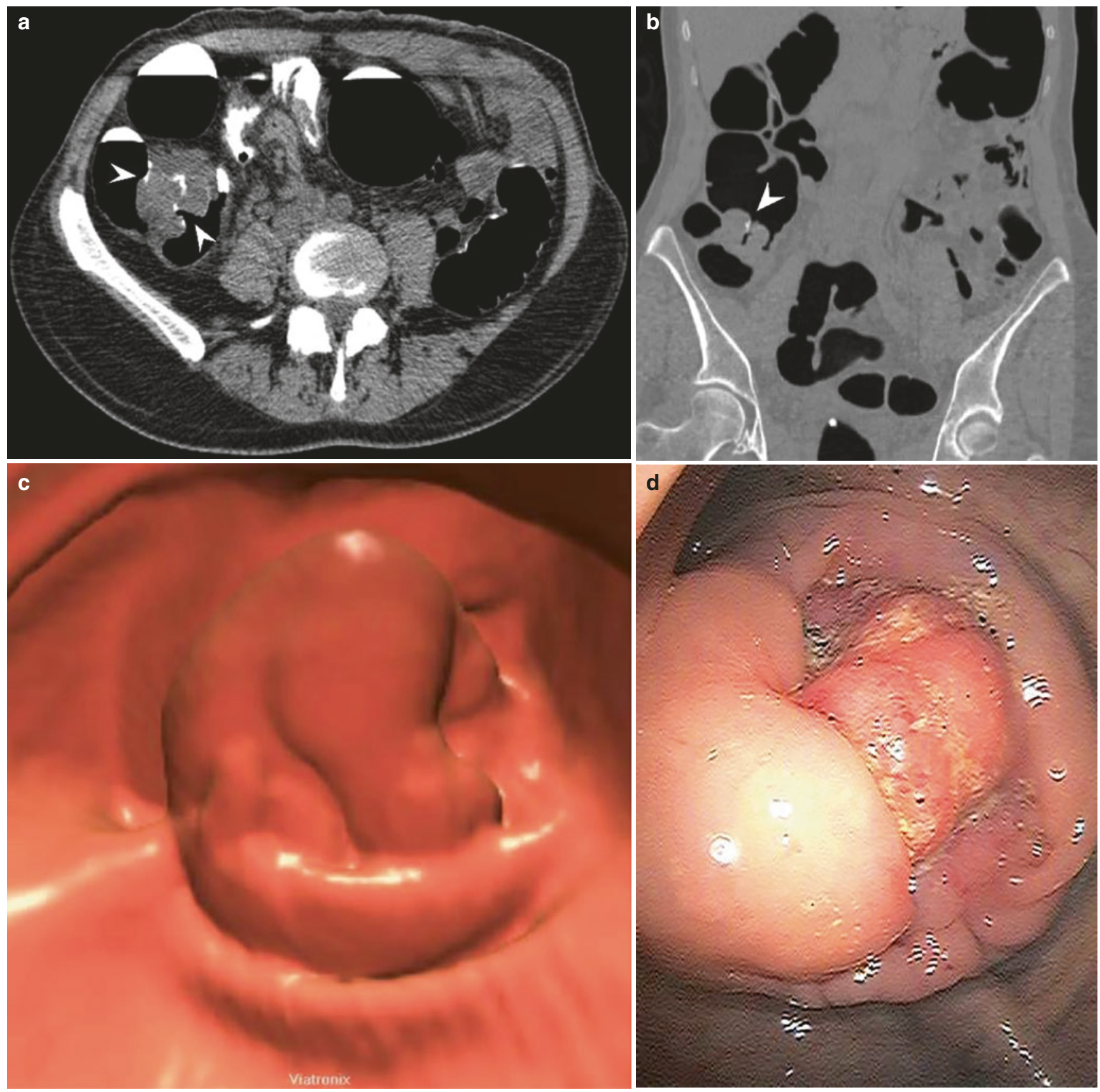

Fig. 16.1 Unsuspected colon cancer in 56-year-old asymptomatic woman undergoing CTC screening. Transverse (a) and coronal (b) 2D images from CTC show a bulky soft tissue mass in the right colon

not require a second procedure-thereby mitigating concerns for redundancy. Those with a high likelihood of polyps may best be triaged to colonoscopy screening.

\subsection{Safety}

The main risk for patients at CTC is the risk of colorectal perforation. The symptomatic perforation rate has been estimated to be 1:20,000 examinations and likely safer among (arrowheads). 3D endoluminal CTC image (c) correlates with the image from subsequent same-day colonoscopy (d)

screening patients [14]. In contrast, colonoscopy perforation rates have been recorded to be in the range of 1:1000 [15], often but not always related to biopsy procedures. Bleeding can also follow colonoscopy intervention and is more common than perforation.

Radiation risk from a CTC examination has been of public concern. It is unfortunate that the risk associated with the low radiation dose required for CTC has been misunderstood. The standard dose at CTC represents about $25-50 \%$ of the dose used for a standard body CT examination. This 
results in an average dose of approximately $2.5-5 \mathrm{mSv}$, similar to annual environmental exposures in many locales that is without known adverse health effects. The doses at CTC can be reduced significantly even further using new noise reduction software (iterative reconstruction). The real risk of this small dose is unknown, but the Health Physics Society has stated that doses in this range are associated with risks for the development of radiation-induced cancer that are either too small to measure or are nonexistent [16]. Even if a very small risk is assumed from radiation exposure at CT, it must be balanced against the risk of developing colon cancer (1:13) and the risks of other alternative procedures including colonoscopy [17].

\subsection{Extracolonic Findings}

Extracolonic abnormalities are common in patients of screening age $[18,19]$. Although lifesaving findings can be identified, most incidental findings are of little clinical significance but can potentially increase the cost of care if additional testing and treatment are recommended. A pragmatic approach to these findings is needed. Radiologists should only recommend follow-up studies for those findings most likely to be of clinical significance. Clinicians will be grateful if additional testing is minimized and for those that might benefit from additional studies to include recommendations for optimal follow-up within the radiologic report.

\subsection{Individual Responsibility}

Maintaining high-quality interpretations is a responsibility that each individual, each practice, and our specialty should assume. The ACR has established a national CTC database within the National Radiology Data Registry (NRDR) [20]. Selected process and outcome metrics can be quickly entered online and compared to national benchmarks. These measures include process metrics related to the $\mathrm{CT}$ technique and the adequacy of patient preparation, outcome metrics related to colon perforation, true positive and false-positive rates for large $(\geq 1 \mathrm{~cm})$ polyps, and the prevalence of significant extracolonic findings. Practices seriously interested in providing the best care should be encouraged to participate in this data registry and manage their practice so benchmark metrics are achieved.

A spirit of cooperation between radiologists and gastroenterologists is needed for optimal patient care. Guidelines will need to be jointly developed for the proper use of colonography and colonoscopy and for processes to efficiently transfer patients with polyps to colonoscopy. Those practices that are able to do this effectively will offer patients a service of high value and will likely find themselves very busy.

Key Point

- CT colonography is a well-validated examination and should be available clinically. Radiologists committed to performing the examination to the highest quality must obtain the education and equipment needed. We have an obligation to educate and to collaborate with referring physicians on the correct use of the technique. We must be vigilant that extracolonic findings are properly reported so that only highly suspicious lesions have recommended additional follow-up testing. Lastly, a commitment to incorporate ongoing quality measures within your daily practice will ensure that patients continue to receive the highest standards of care. Radiology has an exciting opportunity to serve the public and potentially reduces the incidence of a common cancer killer.

\section{References}

1. Siegel R, Naishadham D, Jemal A. Cancer statistics, 2012. CA Cancer J Clin. 2012;62:10-29.

2. Yee J, Hung RK, Akerkar GA, Wall SD. The usefulness of glucagon hydrochloride for colonic distention in CT colonography. AJR. 1999;173:169-72.

3. Laghi A. Computed tomography Colonography in 2014: an update on technique and indications. World $\mathrm{J}$ Gastroenterol. 2014;20:16858-67.

4. Flicek KT, Hara AK, Silva AC, et al. Reducing the radiation dose for CT colonography using adaptive statistical iterative reconstruction: a pilot study. AJR. 2010;195:126-31.

5. Hara AK, Blevins M, Chen MH, et al. ACRIN CT Colonography trial: does reader's preference for primary two-dimensional versus primary three-dimensional interpretation affect performance? Radiology. 2011;259:435-41.

6. Pickhardt PJ, Choi JR, Hwang I, et al. Computed tomographic virtual colonoscopy to screen for colorectal neoplasia in asymptomatic adults. N Engl J Med. 2003;349:2191-200.

7. Johnson CD, Chen MH, Toledano A, et al. Accuracy of CT colonography for detection of large adenomas and cancer. N Engl J Med. 2008;359:1207-17.

8. Fletcher JG, Chen MH, Herman BA, et al. Can radiologist training and testing ensure high performance in CT colonography? Lessons from the National CT Colonography Trial. Am J Roentgenol. 2010 Jul;195(1):117-25.

9. Robbins JB, Kim DH. Computed tomographic Colonography: evidence and techniques for screening. Semin Roentgenol. 2013 Jul;48:264-72.

10. Centers for Disease Control and Prevention. Vital signs: colorectal cancer screening among adults aged 50-75 years-United States, 2008. MMWR Morb Mortal Wkly Rep. 2010:808-12.

11. Beebe TJ, Johnson CD, Stoner SM, Anderson KJ, Limburg PJ. Assessing attitudes toward laxative preparation in colorectal 
cancer screening and effects on future testing: potential receptivity to computed tomographic colonography. Mayo Clin Proc. 2007;82:666-71.

12. Johnson CD, Kriegshauser JS, Lund JT, et al. Partial preparation computed tomographic colonography: a feasibility study. Abdom Imaging. 2011;36:707-12.

13. Johnson CD, Manduca A, Fletcher JG, et al. Noncathartic CT colonography with stool tagging: performance with and without electronic subtraction. Am J Roentgenol. 2006;190:361-6.

14. Pickhardt PJ. Incidence of colonic perforation at CT colonography: review of existing data and implication for screening of asymptomatic adults. Radiology. 2006;239:313-6.

15. Waye JD, Kahn O, Auerbach ME. Complications of colonoscopy and flexible sigmoidoscopy. Gastrointest Endosc Clin N Am. 1996;6:342-77.
16. Radiation risk in perspective. Position Statement of the Health Physics Society July 2010. http://hps.org/documents/risk_ps010-2. pdf

17. Hendee WR, O'Connor MK. Radiation risks of medical imaging: separating fact from fantasy. Radiology. 2012;264:312-21.

18. Gluecker TM, Johnson CD, Wilson LA, et al. Extracolonic findings at CT colonography: evaluation of prevalence and cost in a screening population. Gastroenterology. 2003;124:911-6.

19. Pickhardt PJ, Kim DH, Meiners RJ, et al. Colorectal and extracolonic cancers detected at screening CT colonography in 10,286 asymptomatic adults. Radiology. 2010;255:83-8.

20. American College of Radiology. National Radiology Data Registry for CT Colonography. https://nrdr.acr.org/Portal/CTC/Main/page. aspx

Open Access This chapter is licensed under the terms of the Creative Commons Attribution 4.0 International License (http://creativecommons. org/licenses/by/4.0/), which permits use, sharing, adaptation, distribution and reproduction in any medium or format, as long as you give appropriate credit to the original author(s) and the source, provide a link to the Creative Commons license and indicate if changes were made.

The images or other third party material in this book are included in the book's Creative Commons license, unless indicated otherwise in a credit line to the material. If material is not included in the book's Creative Commons license and your intended use is not permitted by statutory regulation or exceeds the permitted use, you will need to obtain permission directly from the copyright holder. 